\title{
Caracterização espectral de sinais caóticos: resultados analíticos
}

\author{
Daniela Mitie Kato e Marcio Eisencraft
}

\begin{abstract}
Resumo-Nas últimas duas décadas, possibilidades de aplicações práticas de sinais caóticos em telecomunicações têm sido consideradas. Para tanto, é relevante conhecer e controlar as características de correlação e espectro destes sinais. Neste trabalho, faz-se um estudo analítico da seqüência de autocorrelação e da densidade espectral de potência de sinais caóticos de tempo discreto gerados por mapas da família tenda inclinada. Ele complementa artigos recentes dos autores em que estas características foram estudadas apenas de forma numérica.
\end{abstract}

Palavras-Chave-geradores caóticos, comunicação usando caos, análise espectral, banda essencial.

Abstract-On the last two decades, possibilities of practical application of chaotic signals in telecommunication have been considered. Bearing this in mind, it is relevant to know and to control the correlation and spectral characteristics of these signals. In this paper we analytically analyse the autocorrelation sequence and the power spectral density of discrete time chaotic signals generated by skew tent maps. It is a complement of our recent works where these characteristics were numerically studied.

Keywords - chaos generators, chaotic communication, spectral analysis, essential bandwidth.

\section{INTRODUÇÃO}

Um sinal caótico é aperiódico, determinístico e apresenta Dependência Sensível às Condições Iniciais (DSCI). Esta última condição significa que, tomando-se duas condições iniciais muito próximas, ao longo das iterações do mapa, os sinais resultantes vão se separando e o módulo da diferença entre eles após poucas iterações é da ordem de grandeza do próprio sinal. Uma definição mais precisa pode ser encontrada, por exemplo, em [1].

Atualmente, existe um grande número de pesquisas envolvendo aplicações de sinais caóticos nas mais diversas áreas [2]. Na Engenharia de Telecomunicações, essas pesquisas intensificaram-se a partir do trabalho [3]. Veja, por exemplo, [4]-[6] e suas referências. Um exemplo interessante e próximo de uma aplicação prática é no espalhamento espectral por seqüência direta, em que os sinais caóticos podem ser aplicados como seqüências espalhadoras, podendo superar os sistemas convencionais em algumas situações [7].

Devido às propriedades que os definem, costuma-se afirmar, e.g. [4], [5], que os sinais caóticos ocupam uma larga faixa de freqüências, possuem Seqüência de Autocorrelação (SAC) impulsiva e que as seqüências de correlação cruzada entre sinais com diferentes condições iniciais apresentam valores baixos.

Daniela Mitie Kato e Marcio Eisencraft, Escola de Engenharia, Universidade Presbiteriana Mackenzie, São Paulo, Brasil, e-mails: danikato@yahoo.com, marcioft@mackenzie.br.
Apesar da grande quantidade de trabalhos publicados sobre aplicação de caos em comunicação, poucos abordam mais profundamente as características espectrais dos sinais caóticos. Alguns trabalhos, como [8]-[11], mostram gráficos da Densidade Espectral de Potência (DEP) de sinais caóticos gerados por sistemas particulares. $\mathrm{O}$ artigo [12] faz um estudo analítico da SAC de sinais gerados pela família de mapas tenda inclinada, mas não apresenta resultado algum referente à DEP destes sinais.

Os sinais gerados pela família de mapas estudada aqui, a família tenda inclinada, apresentam comportamento rico e variado, mas ainda assim de fácil esquematização. Pelo fato destes mapas serem lineares por partes e suas órbitas apresentarem densidade invariante uniforme, tornam-se mais fáceis os cálculos teóricos e problemas envolvendo estimações [13]. Além disso, nesta família está incluso o mapa tenda, cujos sinais gerados possuem características espectrais conhecidas [13].

Recentemente, os autores apresentaram resultados numéricos referentes à SAC e à DEP de sinais caóticos gerados por esta família [14], [15]. O objetivo do presente artigo é apresentar estes resultados de forma analítica, validando, assim, os resultados obtidos anteriormente.

Este trabalho está organizado da seguinte forma: na Seção II é apresentada a família de mapas tenda inclinada e resumidas suas principais características. Na Seção III são deduzidas a SAC e a DEP das órbitas desta família. Na Seção IV é analisada a relação entre banda essencial e expoente de Lyapunov. Por fim, na Seção V, são expostas as conclusões do trabalho.

\section{A FAmília de Mapas Tenda InClinada}

Um sistema dinâmico unidimensional em tempo discreto é definido pela equação de diferenças

$$
s(n+1)=f(s(n)),
$$

sendo $f: U \rightarrow U, U \subset \mathbb{R}, n \in \mathbb{N}$ e $s(0) \in U$. Para cada condição inicial $s(0)=s_{0}$, uma órbita ou sinal $s\left(n, s_{0}\right)=$ $f^{n}\left(s_{0}\right)$ é definido, em que $f^{n}\left(s_{0}\right)$ é a $n$-ésima iteração do mapa $f(\cdot)$ sobre $s_{0}$. Quando a condição inicial $s_{0}$ estiver subentendida ou não for relevante, $s\left(n, s_{0}\right)$ é representado simplesmente por $s(n)$.

Neste trabalho, foca-se na família de mapas tenda inclinada, uma modificação do mapa proposto em [12]. Um mapa desta família é definido por

$$
s(n+1)=f_{I}(s(n))
$$




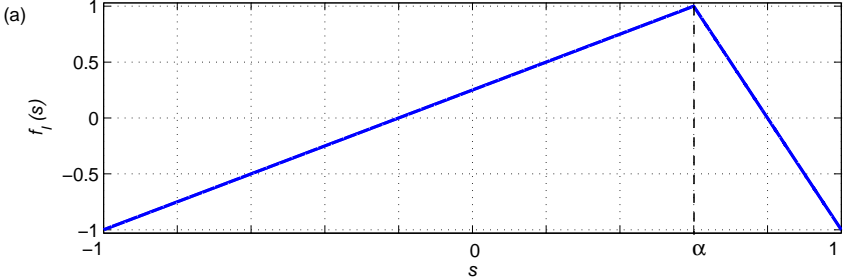

(b)

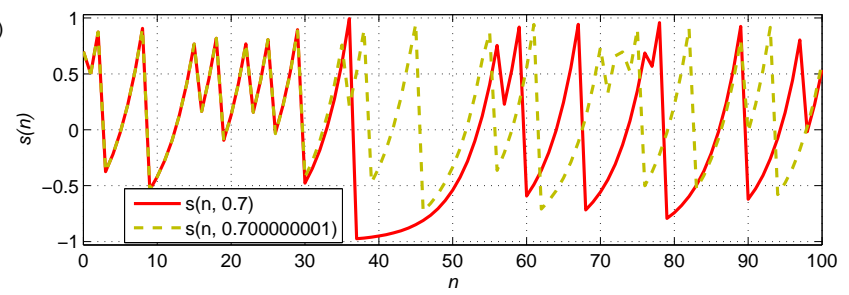

Fig. 1. (a) Mapa tenda inclinada e (b) trechos de órbitas do mapa tenda inclinada com $s_{0}=0.7$ e $s_{0}=0.700000001$.

em que

$$
f_{I}(s)= \begin{cases}\frac{2}{\alpha+1} s+\frac{1-\alpha}{\alpha+1}, & -1<s<\alpha \\ \frac{2}{\alpha-1} s-\frac{\alpha+1}{\alpha-1}, & \alpha \leq s<1\end{cases}
$$

e $\{\alpha, s(0)\} \subset U=(-1,1)$. O parâmetro $\alpha$ é a abscissa em que se localiza o ápice da tenda. Para $\alpha=0$ obtém-se o mapa tenda, que gera órbitas caóticas apenas para condições iniciais irracionais [1].

Na Figura 1(a) é mostrado um exemplo de mapa tenda inclinada para $\alpha=0.6$ e na Figura 1(b), dois trechos de sinal deste mapa com condições iniciais muito próximas, $s_{0}=0.7$ e $s_{0}=0.700000001$. Observa-se que o sinal é aperiódico e apresenta DSCI, características dos sinais caóticos.

A DSCI é geralmente quantificada por meio do expoente de Lyapunov. Este expoente mede a taxa de divergência exponencial média entre duas órbitas muito próximas. Se o valor do expoente é maior que zero, então um sinal aperiódico é caótico [1].

O expoente de Lyapunov $h$ de uma órbita $s\left(n, s_{0}\right)$ é calculado por meio de

$$
h=\lim _{N \rightarrow \infty} \frac{1}{N}\left(\sum_{n=0}^{N-1} \ln \left|f^{\prime}\left(s\left(n, s_{0}\right)\right)\right|\right),
$$

em que $f^{\prime}(s)$ é a derivada de $f(s)$ [1].

Pode-se mostrar [16] que, para as órbitas da família de mapas tenda inclinada, o expoente de Lyapunov $h_{I}$ depende apenas do parâmetro $\alpha$, sendo dado por

$$
h_{I}=\frac{\alpha+1}{2} \ln \left(\frac{2}{\alpha+1}\right)+\frac{1-\alpha}{2} \ln \left(\frac{2}{1-\alpha}\right) .
$$

Na Figura 2 é mostrado um gráfico de $h_{I}$ em função de $\alpha$. Nota-se que, para todos os valores de $\alpha$ admissíveis, $h_{I}>0$, ou seja, os sinais aperiódicos gerados por esta família de mapas são caóticos. O máximo valor de $h_{I}$ é $\ln 2$ para $\alpha=0$.

Em [17] mostra-se que as amostras de quase todos os sinais gerados por estes mapas para qualquer $\alpha$ distribuemse uniformemente no intervalo $[-1,1]$. Assim, sua densidade

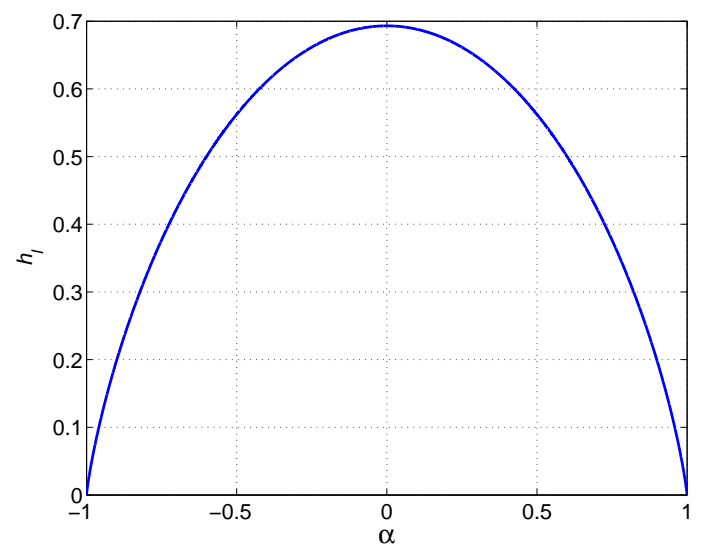

Fig. 2. Expoente de Lyapunov $h_{I}$ de órbitas de $f_{I}(\cdot)$ para $\alpha$ no intervalo $(-1,1)$.

invariante é

$$
p(s)=\frac{1}{2}, \quad-1 \leq s \leq 1 .
$$

Desta forma, eles possuem média nula e potência média

$$
P_{\text {med }}=\frac{1}{3}
$$

para qualquer valor de $\alpha$ [17].

\section{SeqüÊnCIA de Autocorrelação e Densidade ESPECTRAL DE POTÊNCIA}

Os sinais caóticos gerados por um mapa podem ser analisados como funções-amostras de um processo estocástico ergódico [18]. Como descrito em [14], [15], um sinal caótico gerado por $f_{I}(\cdot)$ possui as mesmas características das demais órbitas caóticas geradas por este mesmo mapa. Assim, a cada condição inicial $s_{0}$ que gere uma órbita caótica, associa-se uma função-amostra do processo ergódico definido pelo mapa.

A seguir faz-se o estudo analítico da SAC e da DEP destes sinais.

\section{A. Seqüência de Autocorrelação}

O desenvolvimento a seguir é inspirado no artigo [12].

A SAC $R(k)$ das órbitas de um mapa $f(\cdot)$ da Eq. (1) é definida por

$$
R(k)=E[s(n) s(n+k)],
$$

sendo $k$ um número inteiro que representa o passo da correlação e $n$ qualquer. A esperança matemática $E[\cdot]$ é tomada sobre todas as condições iniciais que geram sinais caóticos. Neste cálculo, considera-se que $s(n)=0$ para $n<0$.

Para facilitar a notação define-se

$$
s(n)=x \quad \text { e } s(n+k)=f_{I}^{k}(x)=y .
$$

Assim, a densidade conjunta $p(x, y)$ é dada por

$$
p(x, y)=p(x) \delta\left(y-f_{I}^{k}(x)\right),
$$




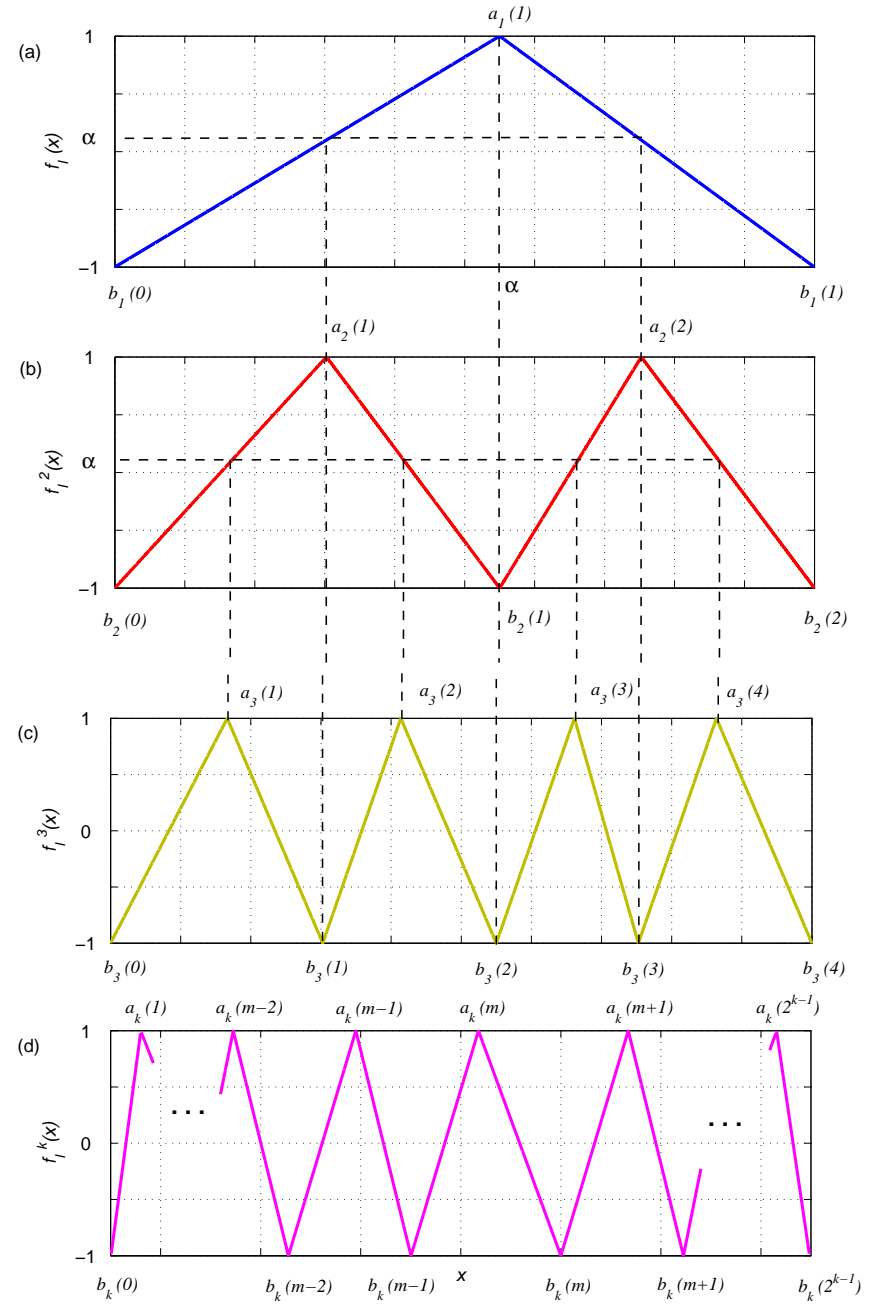

Fig. 3. (a) Mapa tenda inclinada, mapa tenda inclinada após (b) uma iteração, (c) duas iterações e (d) um trecho do mapa tenda inclinada após $k$ iterações.

em que $p(\cdot)$ é a densidade invariante do mapa $f_{I}(\cdot)$ e $\delta(\cdot)$ representa a função delta de Dirac. Desta forma, como $U=$ $[-1,1]$, usando a Eq. (6), tem-se para os mapas $f_{I}(\cdot)$

$$
\begin{aligned}
& R(k)=E[x y]=\int_{-1}^{1} \int_{-1}^{1} x y p(x, y) d x d y \\
& =\int_{-1}^{1} \int_{-1}^{1} x y p(x) \delta\left(y-f_{I}^{k}(x)\right) d x d y=\frac{1}{2} \int_{-1}^{1} x f_{I}^{k}(x) d x
\end{aligned}
$$

O mapa $f_{I}(\cdot)$ é constituído de dois segmentos de reta de inclinações de sinais opostos. A imagem de cada um desses segmentos é igual ao domínio $U$ do mapa. Conseqüentemente, o gráfico $f_{I}^{k}(\cdot)$ consiste de $2^{k}$ segmentos. Na Figura 3(a) é ilustrado o mapa $f_{I}(x)$ para $\alpha=0.1$ e na Figura 3(b), o mesmo mapa após uma iteração, $f_{I}^{2}(x)$. Na Figura 3(c) é apresentado o gráfico de $f_{I}^{3}(x)$ e na Figura 3(d) é mostrado um trecho do gráfico de $f_{I}^{k}(x)$ para um $k$ genérico. Representa-se a $m$-ésima solução da equação $f_{I}^{k}(\cdot)=1$ por $a_{k}(m)$, em que $1 \leq m \leq 2^{k-1}$ e a $m$-ésima solução da equação $f_{I}^{k}(\cdot)=-1$ por $b_{k}(m)$, em que $0 \leq m \leq 2^{k-1}$.

As equações dos segmentos que constituem o mapa $f_{I}^{k}(x)$ são:

- Segmento que passa por $\left(b_{k}(m-1),-1\right)$ e $\left(a_{k}(m), 1\right)$ :

$$
\begin{aligned}
y & =\frac{2}{a_{k}(m)-b_{k}(m-1)}\left(x-b_{k}(m-1)\right)-1 \\
& =\frac{2 x-a_{k}(m)-b_{k}(m-1)}{a_{k}(m)-b_{k}(m-1)} .
\end{aligned}
$$

- Segmento que passa por $\left(a_{k}(m), 1\right)$ e $\left(b_{k}(m),-1\right)$ :

$$
\begin{aligned}
y & =\frac{-2}{b_{k}(m)-a_{k}(m)}\left(x-a_{k}(m)\right)+1 \\
& =\frac{2 x-a_{k}(m)-b_{k}(m)}{a_{k}(m)-b_{k}(m)} .
\end{aligned}
$$

Substituindo-se as Eqs. (12) e (13) na Eq. (11), tem-se

$$
\begin{aligned}
R(k) & =\frac{1}{2} \sum_{m=1}^{2^{k-1}}\left[\int_{b_{k}(m-1)}^{a_{k}(m)} x\left(\frac{2 x-a_{k}(m)-b_{k}(m-1)}{a_{k}(m)-b_{k}(m-1)}\right) d x\right. \\
& \left.+\int_{a_{k}(m)}^{b_{k}(m)} x\left(\frac{2 x-a_{k}(m)-b_{k}(m)}{a_{k}(m)-b_{k}(m)}\right) d x\right]
\end{aligned}
$$

Calculando-se as integrais da Eq. (14) separadamente, obtém-se

$$
\begin{aligned}
& \int_{b_{k}(m-1)}^{a_{k}(m)}\left(\frac{2 x^{2}-\left(a_{k}(m)+b_{k}(m-1)\right) x}{a_{k}(m)-b_{k}(m-1)}\right) d x \\
& =\frac{\left(a_{k}(m)-b_{k}(m-1)\right)^{2}}{6}
\end{aligned}
$$

e

$$
\begin{aligned}
& \int_{a_{k}(m)}^{b_{k}(m)}\left(\frac{2 x^{2}-\left(a_{k}(m)+b_{k}(m)\right) x}{a_{k}(m)-b_{k}(m)}\right) d x \\
& =-\frac{\left(a_{k}(m)-b_{k}(m)\right)^{2}}{6} .
\end{aligned}
$$

Assim,

$R(k)$

$$
=\frac{1}{12} \sum_{m=1}^{2^{k-1}}\left[\left(a_{k}(m)-b_{k}(m-1)\right)^{2}-\left(a_{k}(m)-b_{k}(m)\right)^{2}\right] .
$$

Visando obter uma fórmula mais simples para $R(k)$, buscase uma forma recursiva para $R(k+1)$ em função de $R(k)$.

Ao iterar uma vez o mapa, passa-se de $f_{I}^{k}(x)$ para $f_{I}^{k+1}(x)$, cuja generalização pode ser feita a partir das Figuras 4(a) e 4(b), em que $w$ e $z$ são raízes da equação $f_{I}^{k}(x)=\alpha$.

Para obter $w$ e $z$, substitui-se $y$ por $\alpha$ nas Eqs. (12) e (13) respectivamente e isola-se $x$. Desta forma, obtém-se

$$
w=\frac{\alpha+1}{2}\left(a_{k}(m)-b_{k}(m-1)\right)+b_{k}(m-1)
$$

$\mathrm{e}$

$$
z=\frac{\alpha+1}{2}\left(a_{k}(m)-b_{k}(m)\right)+b_{k}(m) .
$$



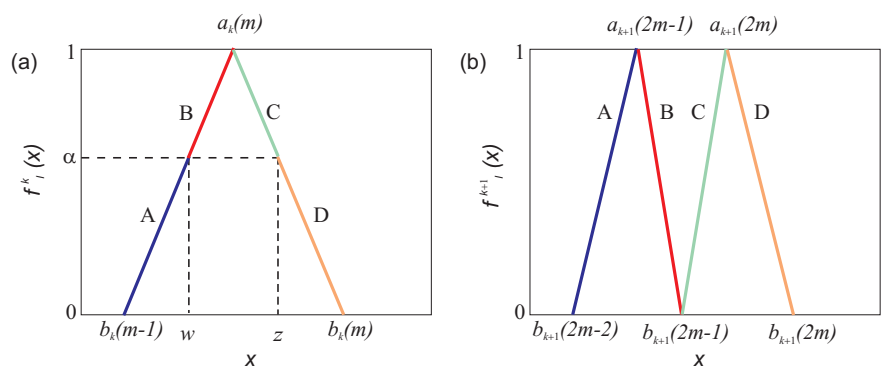

Fig. 4. (a) Trecho do mapa genérico $f_{I}^{k}(\cdot)$ e (b) trecho deste mapa após uma iteração $f_{I}^{k+1}(\cdot)$.

Relacionando-se os gráficos da Figura 4, observa-se que

$$
\begin{aligned}
& b_{k+1}(2 m-2)=b_{k}(m-1) \\
& a_{k+1}(2 m-1)=w \\
& b_{k+1}(2 m-1)=a_{k}(m) \\
& a_{k+1}(2 m)=z \\
& b_{k+1}(2 m)=b_{k}(m) .
\end{aligned}
$$

Calculando-se $R(k+1)$ a partir da Eq. (17), obtem-se

$$
\begin{aligned}
& R(k+1)=\frac{1}{12} \sum_{m=1}^{2^{k}}\left[\left(a_{k+1}(m)-b_{k+1}(m-1)\right)^{2}\right. \\
& \left.-\left(a_{k+1}(m)-b_{k+1}(m)\right)^{2}\right] .
\end{aligned}
$$

Separando-se as parcelas de índices pares e ímpares,

$$
\begin{aligned}
& R(k+1) \\
& \quad=\frac{1}{12} \sum_{m=1}^{2^{k-1}}\left[\left(a_{k+1}(2 m)-b_{k+1}(2 m-1)\right)^{2}-\left(a_{k+1}(2 m)\right.\right. \\
& \left.\quad-b_{k+1}(2 m)\right)^{2}+\left(a_{k+1}(2 m-1)-b_{k+1}(2 m-2)\right)^{2} \\
& \left.\quad-\left(a_{k+1}(2 m-1)-b_{k+1}(2 m-1)\right)^{2}\right] .
\end{aligned}
$$

Em seguida, substituindo-se as Eqs. (18-24) e realizando-se algumas manipulações, chega-se a

$$
\begin{aligned}
& R(k+1) \\
& =\frac{1}{12} \sum_{m=1}^{2^{k+1}}\left\{\left[\frac{\alpha+1}{2}\left(a_{k}(m)-b_{k}(m)\right)+b_{k}(m)-a_{k}(m)\right]^{2}\right. \\
& -\left[\frac{\alpha+1}{2}\left(a_{k}(m)-b_{k}(m)\right)\right]^{2} \\
& +\left[\frac{\alpha+1}{2}\left(a_{k}(m)-b_{k}(m-1)\right)\right]^{2} \\
& \left.-\left[\frac{\alpha+1}{2}\left(a_{k}(m)-b_{k}(m-1)\right)+b_{k}(m-1)-a_{k}(m)\right]^{2}\right\} .
\end{aligned}
$$

Expandindo-se os termos quadráticos do somatório da Eq. (27) e agrupando-se termos comuns, finalmente obtém-se

$$
\begin{aligned}
& R(k+1) \\
& =\frac{\alpha}{12} \sum_{m=1}^{2^{k-1}}\left[\left(a_{k}(m)-b_{k}(m-1)\right)^{2}-\left(a_{k}(m)-b_{k}(m)\right)^{2}\right] .
\end{aligned}
$$

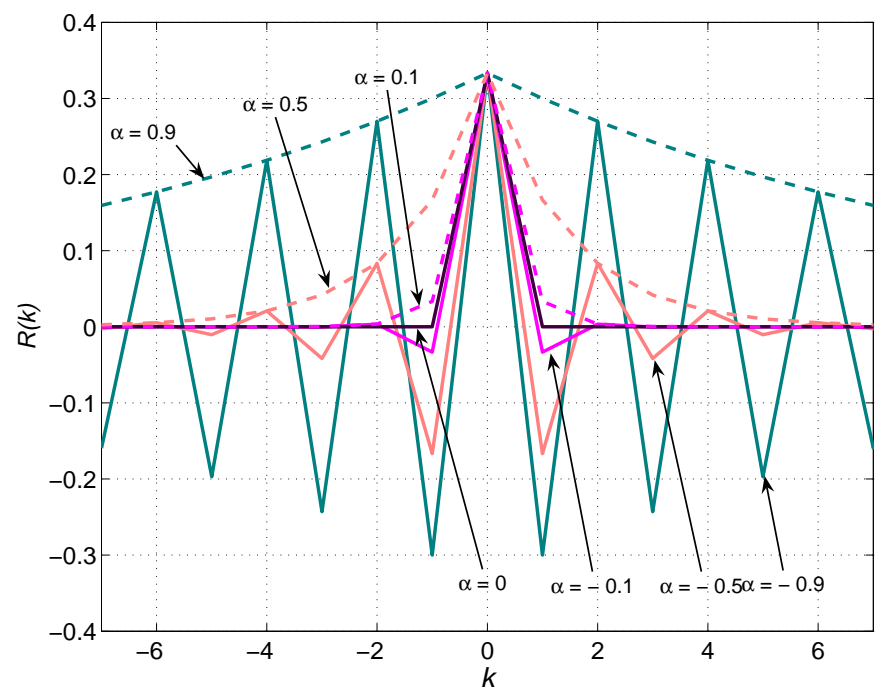

Fig. 5. SAC para sinais do mapa tenda inclinada para alguns valores de $\alpha$.

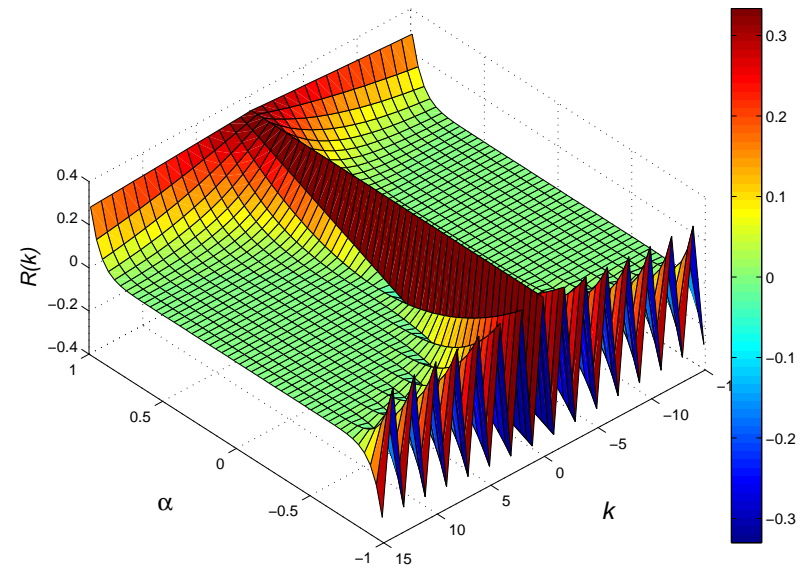

Fig. 6. SAC para sinais do mapa tenda inclinada para valores de $\alpha$ no intervalo $(-1,1)$.

Comparando as Eqs. (17) e (28), observa-se que

$$
R(k+1)=\alpha R(k)
$$

e usando-se a Eq. (6)

$$
\begin{aligned}
R(0) & =E\left[x^{2}\right]=\int_{-1}^{1} x^{2} p(x) d x \\
& =\frac{1}{2} \int_{-1}^{1} x^{2} d x=\left.\frac{1}{2} \frac{x^{3}}{3}\right|_{-1} ^{1}=\frac{1}{3} .
\end{aligned}
$$

Desta forma, resolvendo-se a equação de diferenças dada na Eq. (29) com a condição inicial da Eq. (30), resulta

$$
R(k)=\frac{1}{3} \alpha^{|k|} \text {. }
$$

Na Figura 5 são ilustrados gráficos da SAC para alguns valores de $\alpha$. Na Figura 6 é ilustrado o comportamento geral da SAC para os valores admissíveis de $\alpha$. Vale ressaltar que $k \in \mathbb{Z}$ e as curvas são traçadas em linhas contínuas apenas para facilitar a visualização. 
Observa-se que para $\alpha>0, R(k)$ decai monotonicamente com $|k|$ e que para $\alpha<0, R(k)$ oscila, indicando que, neste caso, para quase quaisquer $n$ e $s_{0}$, as amostras $s\left(n, s_{0}\right)$ e $s\left(n+1, s_{0}\right)$ têm sinais diferentes. Nota-se que, se $\alpha_{1}=-\alpha_{2}$,

$$
R_{\alpha_{2}}(k)=(-1)^{k} R_{\alpha_{1}}(k),
$$

em que $R_{\alpha_{1}}(k)$ é a SAC das órbitas geradas quando $\alpha=\alpha_{1}$ e $R_{\alpha_{2}}(k)$ é a SAC das órbitas geradas quando $\alpha=\alpha_{2}$.

Estes resultados mostram que sinais caóticos nem sempre possuem SAC na forma impulsiva. Neste caso, obtém-se esta forma apenas para $\alpha=0$, coincidindo com o resultado obtido em [13] para $\beta=2$.

\section{B. Análise da Densidade Espectral de Potência}

A DEP $S(\omega)$ é obtida calculando-se a Transformada de Fourier de Tempo Discreto (TFTD) de $R(k)$, considerandose $k$ a variável temporal [20].

Neste caso,

$$
R(k)=\frac{1}{3} \alpha^{|k|}=\frac{1}{3}\left[\alpha^{k} u(k)+\alpha^{-k} u(-(k+1))\right],
$$

em que $u(k)$ representa a função degrau, ou seja,

$$
u(k)=\left\{\begin{array}{c}
1, \quad k \geq 0 \\
0, \quad k<0
\end{array} .\right.
$$

A TFTD de $R(k)$ é dada por

$$
\begin{aligned}
S(\omega) & =\frac{1}{3}\left[\sum_{k=0}^{\infty} \alpha^{k} e^{-j \omega k}+\sum_{k=-\infty}^{-1} \alpha^{-k} e^{-j \omega k}\right] \\
& =\frac{1}{3}\left(\frac{1}{1-\alpha e^{-j \omega}}+\frac{1}{1-\alpha e^{j \omega}}-1\right) \\
& =\frac{1-\alpha^{2}}{3\left(1+\alpha^{2}-2 \alpha \cos (\omega)\right)}
\end{aligned}
$$

Na Figura 7 são mostrados gráficos da DEP para alguns valores de $\alpha$ e na Figura 8 é apresentado, de forma normalizada, o comportamento geral para todos os valores de $\alpha$. Nota-se que são mostrados gráficos apenas para frequiências positivas pelo fato das DEPs, neste caso, serem funções pares.

Pela Eq. (35) e pelos gráficos apresentados, observa-se que o parâmetro da família controla a forma como a potência está distribuída na freqüência. Quanto maior o módulo de $\alpha$, menor a faixa de frequiências em que está concentrada a potência do sinal resultante. Além disso, o sinal de $\alpha$ define se a potência está concentrada nas altas ou baixas freqüências.

Pode-se observar na Figura 7 que as DEPs de sinais gerados por mapas com valores de $\alpha$ opostos apresentam simetria em relação a $\omega=\pi / 2$, ou seja, se $\alpha_{1}=-\alpha_{2}$,

$$
S_{\alpha_{2}}(\omega)=S_{\alpha_{1}}(\omega-\pi)
$$

em que $S_{\alpha_{1}}(\omega)$ é a DEP das órbitas geradas quando $\alpha=\alpha_{1}$ e $S_{\alpha_{2}}(\omega)$ é a DEP das órbitas geradas quando $\alpha=\alpha_{2}$.

Este deslocamento é esperado devido a relação dada na Eq. (32). Quando um sinal $s(n)$ é multiplicado por um $e^{j \omega_{0} k}$, sua TFTD é $S\left(\omega+\omega_{0}\right)$ [20]. Como $(-1)^{k}=\left(e^{-j \pi}\right)^{k}$, levando-se em consideração a Eq. (32), conclui-se que a DEP para valores opostos de $\alpha$ estarão deslocados de $\pi$.

Estes resultados podem ser melhor quantificados utilizandose o conceito de banda essencial.

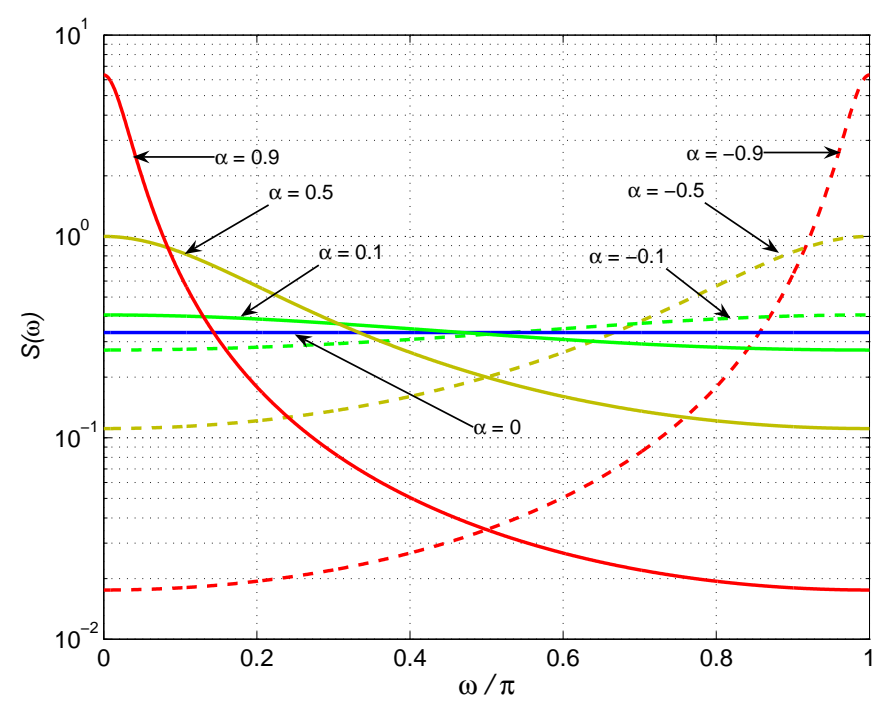

Fig. 7. DEP para sinais do mapa tenda inclinada para alguns valores de $\alpha$.

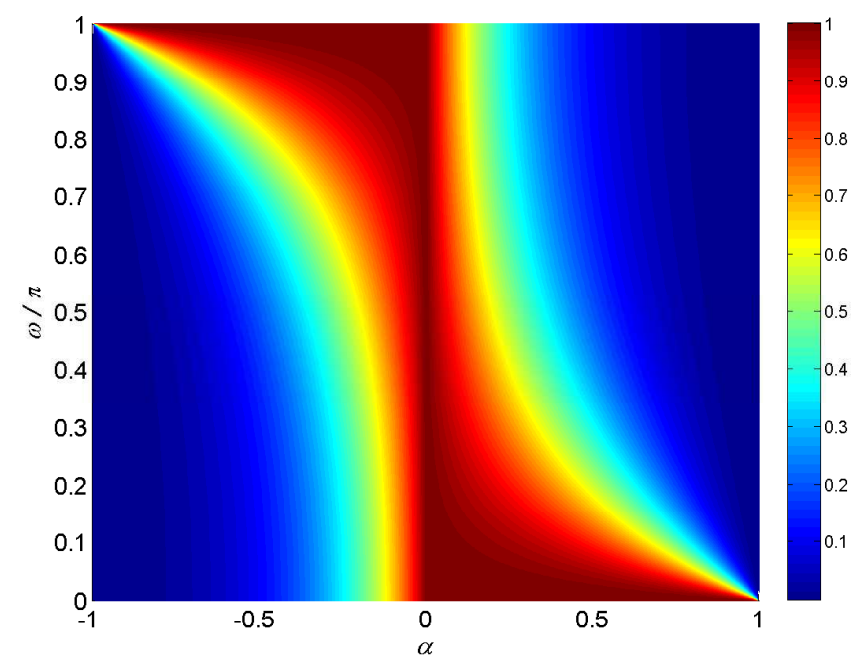

Fig. 8. Comportamento geral da DEP normalizada para os valores admissíveis de $\alpha$.

\section{BANDA ESSENCIAL}

A banda essencial $B$ é definida como o comprimento do intervalo de freqüência em que uma porcentagem $p$ da potência do sinal está concentrada. Aqui, utiliza-se $p=95 \%$ como é usual na literatura de telecomunicações [18].

Para calcular $B$ de um sinal cuja potência está concentrada nas baixas freqüências, integra-se a DEP do sinal no trecho em que uma fração $p$ do sinal esteja concentrada, ou seja,

$$
\int_{0}^{B} S(\omega) d \omega=p \int_{0}^{\pi} S(\omega) d \omega .
$$

Pode-se observar que para valores de $\alpha$ opostos, devido às simetrias ilustradas na Figura 7 e justificadas na seção anterior, a banda essencial dos sinais gerados são iguais. Assim, no desenvolvimento seguinte, considera-se apenas valores positivos de $\alpha$. 


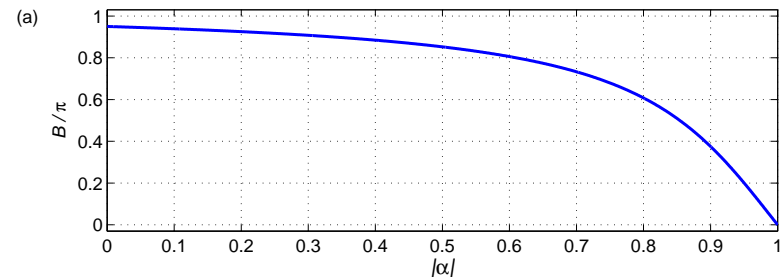

(b)

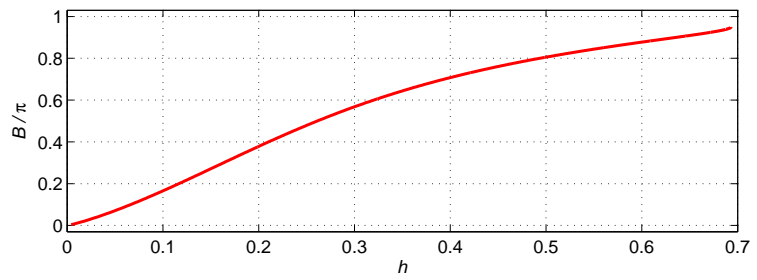

Fig. 9. Banda essencial em função (a) de $|\alpha|$ e (b) do expoente de Lyapunov.

Utilizando-se o teorema de Parseval [20] e a Eq. (7), tem-se

$$
\frac{1}{\pi} \int_{0}^{\pi} S(\omega) d \omega=P_{\text {med }}=\frac{1}{3}
$$

ou

$$
\int_{0}^{\pi} S(\omega) d \omega=\frac{\pi}{3}
$$

Utilizando-se a Eq. (35), obtém-se

$$
\int_{0}^{B} S(\omega) d \omega=\frac{2}{3} \arctan \left[\left|\frac{\alpha+1}{\alpha-1}\right| \tan \left(\frac{B}{2}\right)\right] .
$$

Substituindo-se as Eqs. (39) e (40) na Eq. (37) e isolando-se $B$, obtém-se

$$
B=2 \arctan \left[\frac{\tan \left(\frac{p \pi}{2}\right)}{\left|\frac{\alpha+1}{\alpha-1}\right|}\right]=2 \arctan \left[\tan \left(\frac{p \pi}{2}\right)\left|\frac{\alpha-1}{\alpha+1}\right|\right] .
$$

A Figura 9 mostra gráficos de $B$ em função do módulo de $\alpha$ e em função do expoente de Lyapunov $h_{I}$ da Eq. (5). Como $B=p \pi$ para $\alpha=0$ e levando-se em consideração a Eq. (6), obtém-se, neste caso, um processo ruído branco uniforme. Já para $\alpha \approx 1$, as órbitas são funções-amostras de um processo de banda extremamente estreita. É importante ressaltar que, em todos os casos, o expoente de Lyapunov é positivo e assim, as funções-amostras aperiódicas destes processos são sempre sinais caóticos para todos os valores de $\alpha$.

Deste modo, escolhendo-se um $\alpha$ adequado, pode-se obter um sinal caótico banda larga ou estreita, com sua potência concentrada nas freqüências altas ou baixas e com a banda essencial bem definida.

Estes resultados comprovam os resultados numéricos apresentados em [14], [15], ou seja, sinais caóticos não implicam banda larga e SAC impulsiva. Por isso é importante estudar mais profundamente o espectro desses sinais pensando em aplicá-los em Telecomunicações.

\section{CONClusões}

Com a realização deste trabalho, confirmou-se analiticamente a possibilidade de se gerar sinais caóticos com banda essencial bem definida, como descrito nos artigos [14], [15], em que estes resultados foram obtidos por meio de simulações computacionais.

Comprovou-se também que o parâmetro $\alpha$ e o expoente de Lyapunov estão fortemente relacionados com a banda essencial $B$. Tendo-se uma banda essencial desejada, por meio da Eq. (41) pode-se obter $\alpha$ e, conseqüentemente, um mapa linear por partes associado que gere esta órbita.

Com estes resultados aumenta-se a possibilidade de aplicações de sinais caóticos em sistemas de comunicação, por exemplo em modulação e multiplexação, como citado em [14], [15]. Em Telecomunicações é fundamental conhecer a banda essencial dos sinais utilizados e por este motivo é relevante expandir estes estudos para sinais caóticos gerados por diversos outros mapas, incluindo-se os multidimensionais.

\section{REFERÊNCIAS}

[1] K. T. Alligood, T. D. Sauer and J. A. Yorke, Chaos - an introduction to dynamical systems. Springer, 1996.

[2] S. H. Strogatz, Nonlinear dynamics and chaos with applications to physics, biology, chemistry and engineering. Addinson-Wesley, 1998.

[3] L. M. Pecora and T. L. Carroll, "Synchronization in chaotic systems," Physical Review Letters, v. 64, n. 8, p. 821-824, Februaty 1990.

[4] M. P. Kennedy, R. Rovatti and G. Setti, Chaotic electronics in telecommunications. CRC Press, 2000.

[5] P. Stavroulakis, Chaos applications in telecommunications. CRC Press, 2005

[6] W. M. Tam, F. C. M. Lau and C. K. Tse, Digital communications with chaos - multiple access techniques and performance. Elsevier, 2007.

[7] R. Rovatti, G. Mazzini and G. Setti, "On the ultimate limits of chaosbased asynchronous DS-CDMA-I: basic definitions and results," IEEE Transactions on Circuits and Systems - I, v. 51, n. 7, p. 1336-1347, July 2004.

[8] T. Matsumoto, L. O. Chua and M. Komuro, "The double scroll," IEEE Transactions on Circuits and Systems - I, v. 32, n. 8, p. 798-817, August 1985.

[9] K. M. Short, "Steps toward unmasking secure communications," International Journal of Bifurcation and Chaos, v. 4, n. 4, p. 959-977, February 1994.

[10] A. C. Faleiros, W. J. Perrella, T. N. Rabello, A. S. Santos and N. Y. Soma, In: P. Stavroulakis, Chaos applications in telecommunications. CRC Press, 2005.

[11] K. Ullmann and I. L. Caldas, "Transitions in the parameter space of a periodically forced dissipative system," Chaos, Solitons \& Fractals, v. 7, n. 11, p. 1913-1921, November 1996.

[12] H. Sakai and H. Tokumaru, "Autocorrelations of a certain chaos," IEEE Transactions on Acoustics, Speech, and Signal Processing, v. 28, n. 5 , p. 588-590, October 1980 .

[13] H. C. Papadopoulos and G. W. Wornell, "Maximum Likelihood Estimation of a Class of Chaotic Signals," IEEE Transactions on Information Theory, v. 41, n. 1, p. 312-317, January 1995.

[14] D. M. Kato and M. Eisencraft, "On the power spectral density of chaotic signals generated by skew tent maps," ISSCS07 - International Symposium on Signals, Circuits and Systems, 2007, v. 1, p. 105-108, July 2007.

[15] D. M. Kato e M. Eisencraft, "Caracterização espectral de sinais caóticos," SBrT07 - XXV Simpósio Brasileiro de Telecomunicações, CDROM, Setembro 2007.

[16] A. Kisel, H. Dedieu and T. Schimming, "Maximum likelihood approaches for noncoherent communications with chaotic carriers," IEEE Transactions Circuits and Systems - I, v. 48, n. 5, p. 533-542, May 2001.

[17] M. Eisencraft, Contribuições da teoria da estimação para modulações digitais que utilizam sinais caóticos. Tese (Doutorado em Engenharia Elétrica) - Escola Politécnica da Universidade de São Paulo. São Paulo, 2006.

[18] B. P. Lathi, Modern digital and analog communication systems. Oxford University Press, 1998.

[19] A. Lasota and M. Mackey, Probabilistic properties of deterministic systems. Cambridge University, 1985.

[20] A. V. Oppenheim, R. W. Schafer and J. R. Buck, Discrete-time signal processing. Prentice-Hall, 1999. 